Rev Méd Urug 2018; 34(2):89-95

http://www.rmu.org.uy/revista/2018v2/art4.pdf

doi:10.29193/RMU.34.2.3

\title{
Calidad oncológica en cirugía del cáncer de recto
}

Javier Chinelli*, Juan Martín Costa†, Macarena Muto‡, Viviana Escobar§, Gustavo Rodríguez Temesio"

\section{Resumen}

Introducción: el pronóstico del cáncer de recto depende, entre otros factores, de la calidad oncológica de la resección quirúrgica, entre los que se deben incluir la correcta disección del mesorrecto y un adecuado número de ganglios linfáticos.

Objetivos: describir los principales factores que determinan la calidad de la cirugía de resección por cáncer de recto en la Clínica Quirúrgica 2 del Hospital Maciel.

Pacientes y método: 36 pacientes operados de coordinación en el período 2012 a 2016.

Resultados: la disección mesorrectal fue completa en el $60 \%$ de los casos, y en más del $50 \%$ se obtuvo un número aceptable de ganglios linfáticos.

Discusión: la calidad oncológica de la cirugía por cáncer de recto depende de mútiples factores, entre los que destacamos el volumen anual de cirugías, la experiencia del equipo, factores del paciente (sexo, índice de masa corporal) y de la enfermedad (topografía baja, estadio, respuesta a la neoadyuvancia).

Conclusiones: los resultados obtenidos pueden considerarse oncológicamente satisfactorios.

Palabras clave: CÁNCER DE RECTO

ESCISIÓN MESORRECTAL

Key words: $\quad$ RECTUM CANCER

MESO-RECTUM DISSECTION

\footnotetext{
*Asistente de Clínica Quirúrgica 2. Hospital Maciel. Montevideo, Uruguay.

† Prof. Adj. de Clínica Quirúrgica 2. Hospital Maciel. Montevideo, Uruguay.

¥ Residente de Clínica Quirúrgica 2. Hospital Maciel. Montevideo, Uruguay.

$\S$ Asistente del Departamento de Anatomía Patológica. Montevideo, Uruguay.

II Prof. Titular de Clínica Quirúrgica 2. Hospital Maciel Montevideo, Uruguay.

Clínica Quirúrgica 2. Departamento de Anatomía Patológica Hospital Maciel. Montevideo, Uruguay.

Los autores declaran no tener conflicto de intereses.

Correspondencia: Dr. Javier Chinelli. Mercedes 1472/402. Montevideo. Uruguay. Correo electrónico: Jchinelli01@hotmail.com

Recibido: $11 / 12 / 18$

Aprobado: 16/2/18.
} 


\section{Introducción}

El tratamiento del cáncer de recto requiere de una integración multidisciplinaria, teniendo en cuenta que se trata de una patología cuyo enfoque diagnóstico y terapéutico ha cambiado en forma radical en los últimos años, sobre todo en los tumores ubicados por debajo de la reflexión peritoneal. En ese sentido, se destacan la introducción de la terapia neoadyuvante, el concepto de escisión mesorrectal total (TME, por su sigla en inglés), así como la mejor comprensión de los factores histopatológicos determinantes del pronóstico oncológico final.

Hasta fines de la década de 1970, la tasa de recidiva locorregional en los pacientes operados por cáncer de recto era elevada, cercana a $40 \%{ }^{(1)}$. En 1979 , Enker señala la necesidad de una resección que vaya más allá del recto, incluyendo una correcta linfadenectomía ${ }^{(2)}$. Ese mismo año, Heald ${ }^{(3)}$ introduce el concepto de la TME, que revolucionó la cirugía del cáncer de recto, lo que implica un cambio en la técnica quirúrgica, que debe resecar el recto junto con el tejido graso perirrectal y la fascia mesorrectal (endopélvica) como un bloque único. El mismo autor publica algunos años después resultados ampliamente satisfactorios, con una tasa de recidiva local de solo $5 \%{ }^{(4)}$.

Por otra parte, la terapia neoadyuvante es actualmente el tratamiento estándar de inicio en los tumores avanzados de recto subperitoneal, dado que la misma, seguida de una TME, ha logrado reducir la tasa de recidiva locorregional mucho más que la TME sola ${ }^{(5)}$.

El resultado oncológico del tratamiento del cáncer de recto está determinado en gran medida por la calidad de la resección quirúrgica y la presencia de factores histopatológicos determinantes, entre los que se destacan la calidad de la disección mesorrectal, el margen de resección circunferencial, el margen distal, el número de ganglios resecados y su afectación, el grado de regresión si es que se efectuó neoadyuvancia, así como otros cuyo significado pronóstico no se encuentra aún bien dilucidado. Está demostrado también que la calidad de la cirugía efectuada no descansa únicamente en la experiencia y competencia del cirujano, sino que esta puede verse afectada por variables propias del paciente y su enfermedad, como la presencia de obesidad o desnutrición, el sexo masculino, la pelvis estrecha, el antecedente de cirugía abdomino-pélvica y el tratamiento neoadyuvante.

Nuestro objetivo es evaluar la calidad de la cirugía del cáncer de recto revisando el estudio anatomopatológico de las piezas de resección en una serie de pacientes operados de coordinación en nuestro servicio de cirugía, identificando las principales variables de impacto pronóstico.

\section{Pacientes y método}

Estudio retrospectivo, observacional y descriptivo.

Criterios de inclusión: pacientes asistidos en la Clínica Quirúrgica 2 del Hospital Maciel en el período comprendido entre los años 2012 y 2016 con diagnóstico presuntivo o confirmado de cáncer rectal (incluidos los tumores de la unión recto-sigmoidea) que fueron sometidos a resección quirúrgica de coordinación. Se excluyen los pacientes operados de urgencia y aquellos tratados mediante resección local.

La información fue obtenida a partir de una base de datos de registro prospectivo de la Unidad de Coloproctología y de la revisión de los informes anatomopatológicos del Departamento de Anatomía Patológica.

Una vez recibida la pieza de resección, el patólogo mide el largo y diámetro del recto, y procede a una evaluación inicial de la integridad del mesorrecto, fotografiando las caras anterior y posterior, para luego entintar toda la superficie no peritonizada del meso (figura 1). Se realiza la apertura longitudinal por su sector anterior desde el cabo proximal hasta $2 \mathrm{~cm}$ por encima del tumor, registrando los márgenes proximal y distal así como la topografía con respecto a la reflexión peritoneal. Se fija la pieza en formol/formalina durante 24 horas, y luego se secciona transversalmente a nivel del tumor, en cortes realizados cada 3-5 $\mathrm{mm}$, que serán posicionados y fotografiados de modo que el más proximal se ubique a la izquierda y arriba, y el más distal abajo y a la derecha. Estos cortes transversales serán examinados al microscopio con la finalidad de evaluar el margen de resección circunferencial, invasión venosa extramural y adenopatías metastásicas mesorrectales. Finalmente se disecan y contabilizan todos los ganglios linfáticos identificables.

Para el análisis del estudio anatomopatológico se siguió el protocolo del Colegio Americano de Patólogos para pacientes con carcinoma primitivo colorectal ${ }^{(6)}$, tomando en cuenta las siguientes variables: vía de abordaje, tamaño tumoral, perforación macroscópica, integridad mesorrectal, tipo histológico, invasión parietal, número de ganglios obtenidos, márgenes distal y circunferencial, respuesta a la neoadyuvancia, invasión linfovascular y neural, depósitos tumorales.

Con respecto a la variable integridad del mesorrecto (tabla 1), se define la misma como:

a) Completa (o plano mesorrectal): mesorrecto intacto o con mínima irregularidad, con defectos que no sobrepasan los $5 \mathrm{~mm}$, sin efecto de cono hacia el margen distal de resección.

b) Casi completa (plano intramesorrectal): uno o más defectos cuya profundidad sobrepasa los $5 \mathrm{~mm}$, leve efecto cono, sin muscular propia visible. 
c) Incompleta (plano de la muscular propia): efecto cono moderado o marcado, exposición de la capa muscular propia.

Los datos fueron recogidos en una planilla Excel.

\section{Resultados}

Fueron incluidos un total de 36 pacientes, 21 de sexo femenino y 15 masculino.

Ocho pacientes fueron operados por vía laparoscópica y 28 por vía laparotómica. El tamaño promedio de las lesiones fue de $4,4 \mathrm{~cm}$, con un rango de $1-15 \mathrm{~cm}$ y no se evidenció perforación macroscópica en ninguno de los casos.

En 22 pacientes (61\%) se constató la integridad del mesorrecto, nueve presentaron algún defecto que fue catalogado como disección mesorrectal parcialmente completa en un caso (3\%) e incompleta en los ocho restantes (22\%). Finalmente, en cinco casos (14\%) este dato no fue consignado en el informe anatomopatológico.

La histología reveló que se trataba de un adenocarcinoma en 31 pacientes $(86 \%)$, de los cuales 28 eran moderadamente diferenciados, uno bien diferenciado y dos pobremente diferenciados. Estenosis inflamatoria en un paciente $(3 \%)$, carcinoma epidermoide en un paciente (3\%), lesión ulcerada sin evidencia de tumor residual en un paciente $(3 \%)$ y adenoma túbulo-velloso en un caso $(3 \%)$.

La invasión en profundidad de las lesiones que finalmente fueron confirmadas como cáncer rectal $(n=34)$ fue la siguiente: pT0 (carcinoma in situ), dos pacientes (6\%); pT1 (invasión hasta la submucosa), dos pacientes (6\%); pT2 (invasión de la capa muscular), nueve pacientes (26\%); pT3 (invasión del mesorrecto), 18 pacientes (53\%); pT4 (invasión de estructuras vecinas), dos pacientes $(6 \%)$. En un paciente (3\%) no se reconoció tumor residual, probablemente debido a una respuesta completa a la neoadyuvancia, por lo que debe catalogarse como ypTx (ypRC).

El número promedio de ganglios linfáticos obtenidos en las piezas de resección fue 14, con un rango muy variable que fue de 2 a 66 ganglios. En 18 pacientes (50\%) el número de ganglios resecados fue mayor a 12 , considerado el mínimo aceptable para lograr una correcta estadificación.

El margen de resección distal fue considerado como comprometido en un caso (3\%) con una distancia de 9 $\mathrm{mm}$ al límite del tumor. Por otra parte, en un paciente (3\%) se informó un margen de resección circunferencial comprometido, menor a $1 \mathrm{~mm}$. En el resto de los casos (94\%) los márgenes fueron libres de compromiso tumoral.
En ocho pacientes se especificó la respuesta a la neoadyuvancia (tabla 2): un caso de respuesta completa (ypTx), dos casos de respuesta parcial, tres casos de no respuesta y dos casos con progresión tumoral a pesar del tratamiento.

Se constataron embolias linfáticas en dos casos $(6 \%)$, vasculares en tres casos (8\%), en tanto que existió invasión perineural en dos casos (6\%), y se observó la presencia de depósitos tumorales extranodales en un paciente (3\%).

\section{Discusión}

La serie comprende 36 pacientes en un período de cuatro años, presentándose la mayoría con tumores localmente avanzados. En el $60 \%$ de los casos la resección mesorrectal fue completa.

La decisión de no incluir a pacientes operados en la urgencia se basa en que la misma persigue un objetivo primario que no es la curación de la enfermedad, sino solucionar una complicación. Posiblemente constituye un sesgo de selección, por tanto sean excluidos pacientes en los que de antemano se espera una cirugía de peor calidad oncológica.

Con respecto a la elección de la vía de abordaje, desde hace tiempo existe evidencia suficiente para considerar el abordaje laparoscópico como oncológicamente equivalente al abierto para el cáncer de colon. Sin embargo, para el cáncer rectal esta equivalencia no ha sido reconocida sino hasta hace relativamente pocos años ${ }^{(7,8)}$. En nuestra Clínica Quirúrgica el desarrollo de la cirugía colorrectal mínimamente invasiva ha tenido lugar sobre todo en los últimos dos años, lo que explica que solo en ocho de los 36 pacientes esta haya sido la vía de elección inicial, y que particularmente esos pacientes pertenezcan al período de 2014-2017, pero fundamentalmente del último año y medio (7/8 pacientes).

La TME se ha convertido en el gold standard en la cirugía del cáncer rectal, siendo clave tanto la integridad del mesorrecto como un margen circunferencial adecuado, ya que ambos han demostrado reducir las tasas de recidiva local ${ }^{(8,9)}$. La disección progresa en un plano avascular, areolar, entre la fascia pélvica parietal y la fascia mesorrectal. Ciertos factores pueden influir en la calidad de la pieza obtenida, bien porque aumentan el grado de dificultad de la cirugía, como la pelvis estrecha androide en el hombre, la cirugía prostática previa, la fibrosis posradioterapia, la obesidad, o bien porque disminuyen la cantidad de tejido graso haciendo aun más cercana la distancia entre el tumor y la fascia mesorrectal, como se ve en pacientes desnutridos. Un estudio multicéntrico belga observó en el análisis multivariado que solamente el índice de masa corporal, la ausencia de downstaging y la cirugía laparoscópica se asociaron con una mayor in- 
cidencia de disecciones casi completas o incompletas ${ }^{(10)}$. El número de pacientes en nuestra serie es muy reducido como para inferir si la presencia de alguno de los factores reconocidos anteriormente mencionados fue determinante o si al menos existió una asociación entre ellos y la presencia de un mesorrecto incompleto. Sin embargo, debemos notar que de esos nueve pacientes en los que existió algún defecto en la integridad de la fascia mesorrectal, en cuatro casos se constató la ausencia de respuesta a la neoadyuvancia y en un caso la progresión bajo tratamiento.

Para realizar la estadificación se emplea el sistema TNM de la American Joint Committee on Cancer (AJCC $)^{(11)}$, sabiendo que la profundidad de la invasión tumoral es un factor pronóstico independiente, y particularmente entre los tumores T3 cuando se extienden más de $5 \mathrm{~mm}$ en el mesorrecto ${ }^{(12)}$. En nuestra serie más de la mitad de los pacientes fueron catalogados como pT3 en el estudio anatomopatológico.

Con respecto a la linfadenectomía, se considera que la misma debe incluir un mínimo de 12 ganglios para una correcta estadificación, ya que en algunos estudios ha demostrado mejorar la sobrevida total con independencia de la presencia o ausencia de metástasis a nivel de los mismos ${ }^{(13)}$. Sin embargo, el ensayo clínico COST de la Clínica Mayo no encuentra una correlación entre el número de ganglios resecados y la sobrevida ${ }^{(14)}$, por lo que el debate acerca del número mínimo aceptable de ganglios permanece hasta el día de hoy. Una alternativa propuesta para determinar el significado pronóstico del estado ganglionar, sobre todo cuando el número total de ganglios obtenidos es menor a 12, es el cálculo del cociente entre ganglios metastásicos y ganglios resecados o MLNR ${ }^{(15)}$ (Metastatic lymph node ratio). Este cobraría especial valor en los pacientes tratados con neoadyuvancia, ya que la misma ha demostrado disminuir el recuento ganglionar posoperatorio. Sin embargo, el principal problema sigue siendo la ausencia de un punto de corte bien establecido para el MLNR, que oscila entre 0,19 y $0,67^{(16)}$. El número de ganglios presentes en el mesorrecto ha sido objeto de estudio en nuestro país, concluyéndose que hay algo más de ocho y que estos se encuentran en su mayoría próximos a la división de la arteria hemorroidal superior ${ }^{(17)}$. En los pacientes de nuestra serie se destaca una importante variabilidad respecto al número de ganglios reclutados en las piezas, señalando, sin embargo, que en la mitad de los casos el mismo resultó oncológicamente satisfactorio (> 12). Pero especialmente importante es tener en cuenta que de los 18 pacientes cuya linfadenectomía no fue considerada satisfactoria, 8 (44\%) habían recibido neoadyuvancia previamente.
En un caso se encontraron depósitos tumorales aislados (extranodales); estos suelen ser catalogados como N1c, ya que se consideran adenopatías metastásicas en las que el tejido linfoganglionar ha sido erradicado por la neoadyuvancia, persistiendo entonces solamente las células tumorales residuales. Sin embargo, se sabe también que la radioterapia puede fragmentar el tumor y dar origen a estos depósitos aislados que no guardan relación con la masa tumoral principal, lo cual puede llevar al error diagnóstico y así catalogar como $\mathrm{N}+$ un paciente que no lo es, y por tanto exponerlo innecesariamente a un tratamiento sistémico posterior y su eventual toxici$\operatorname{dad}^{(18)}$.

Al momento de analizar el margen de resección distal deben considerarse dos elementos: la difusión tumoral discontinua a través de linfáticos del mesorrecto y el crecimiento continuo intra y extramural. Con respecto a la primera, es la que posibilita la presencia de depósitos tumorales distales a más de $2 \mathrm{~cm}$ de la lesión, por lo que en tumores del recto subperitoneal la ETM hasta el plano de los elevadores es necesaria, y en tumores por encima de la reflexión la ETM deberá extenderse $5 \mathrm{~cm}$ distal al tumor. $Y$ en cuanto a la segunda, solo en 3,6\% de los casos la extensión continua supera los $2 \mathrm{~cm}^{(19)}$, por lo que en tumores subperitoneales un margen distal aceptado es entre 0,5 y $2 \mathrm{~cm}$. También debemos mencionar que los márgenes se determinan una vez estudiados los anillos de la sutura, e idealmente antes de la fijación, ya que esta determina un acortamiento significativo de la pie$\mathrm{za}^{(20)}$. En nuestra serie, todos los pacientes presentaron márgenes distales dentro de este rango.

El margen de resección circunferencial (MRC), que corresponde a la distancia entre la fascia mesorrectal y el punto de mayor invasión en profundidad del tumor, se considera negativo si es mayor a $1 \mathrm{~mm}$, en cuyo caso predice menor índice de recidiva local ${ }^{(21)}$. Por el contrario, de ser positivo aumenta 3,5 veces el riesgo de recidiva y duplica la mortalidad específica por la enferme$\mathrm{dad}^{(9)}$. El compromiso del MRC puede ser por extensión directa del tumor o depósitos tumorales, adenopatías metastásicas, invasión linfo-vascular o perineural. Sin embargo, el MRC por sí solo no es un indicador de la calidad oncológica de la cirugía, ya que el mismo depende de varios factores entre los que se destacan la extensión en profundidad del tumor ( $\mathrm{T}$ ), la topografía en recto bajo, la amputación abdómino-perineal y la diferenciación. De hecho, una cirugía puede haber sido R0 aun con un MRC positivo. En la serie se presentó solamente un paciente con MRC positivo, tratándose de un tumor pT3 de $5 \mathrm{~cm}$ de tamaño, subperitoneal, en contacto con la fascia mesorrectal (completa), en el que se optó por una resección anterior baja laparoscópica. 


\begin{tabular}{|lc|}
\hline \multicolumn{2}{l}{ Tabla 1. Disección mesorrectal. } \\
\hline Integridad del mesorrecto & $N(\%)$ \\
\hline Completa & $22(61 \%)$ \\
Casi completa & $1(3 \%)$ \\
Incompleta & $8(22 \%)$ \\
No consignado & $5(14 \%)$ \\
\hline
\end{tabular}

Otra de las variables de mayor impacto pronóstico es sin dudas la respuesta histopatológica a la neoadyuvancia. En este sentido se han propuesto múltiples formas de evaluarla, siendo una de ellas el esquema de Ryan modificado (tabla 2), el cual ha demostrado buena concordancia interobservador ${ }^{(22)}$. Debemos mencionar que una de las debilidades de este trabajo ha sido la escasa referencia al grado de regresión tumoral en los informes anatomopatológicos, el cual solamente fue detallado en ocho pacientes, con un caso de respuesta completa (урТх), dos casos de respuesta parcial, tres de no respuesta y dos casos con progresión tumoral bajo neoadyuvancia. Es de capital importancia que el patólogo reciba la pieza con toda la información necesaria, incluyendo si el paciente recibió neoadyuvancia, ya que muchas veces este dato no fue consignado y por ende no se tuvo en cuenta al momento del examen microscópico.

Finalmente, la invasión perineural y linfovascular extramural han demostrado también ser indicadores pronósticos y relacionarse con un mayor riesgo de reci-
Tabla 2. Grado de respuesta a la neoadyuvancia según esquema de Ryan modificado.

\begin{tabular}{lcc}
\hline Regresión & Score & $n$ \\
\hline Ausencia de células neoplásicas viables & 0 & 1 \\
$\begin{array}{l}\text { Células aisladas o grupos aislados (respuesta } \\
\text { casi completa) }\end{array}$ & 1 & 2 \\
$\begin{array}{l}\text { Tumor residual pero con regresión evidente } \\
\text { (respuesta parcial) }\end{array}$ & 2 & 3 \\
$\begin{array}{l}\text { Tumor extenso sin regresión (respuesta pobre / } \\
\text { no respuesta) }\end{array}$ & 3 & 2 \\
\hline
\end{tabular}

diva y menor sobrevida ${ }^{(23-25)}$. La invasión perineural tiene el inconveniente de ser más difícil de detectar, en tanto que la invasión de venas extramurales puede incluso ser consignada en la evaluación preoperatoria mediante resonancia magnética, lo cual debe llevar a una búsqueda exhaustiva de la misma en la pieza de resección.

Un elemento que recientemente ha vuelto a ser considerado como un importante factor pronóstico es el denominado tumor budding, definido como la presencia de células tumorales aisladas o en pequeños grupos de más de cinco células situadas en el frente infiltrante del tumor ${ }^{(26)}$. Cuando este se encuentra en alto grado, se asocia a otros factores de mal pronóstico como son mayor profundidad de invasión, metástasis ganglionares e invasión perineural ${ }^{(27)}$. De acuerdo a las últimas recomendaciones del Colegio Americano de Patólogos ${ }^{(28)}$, este

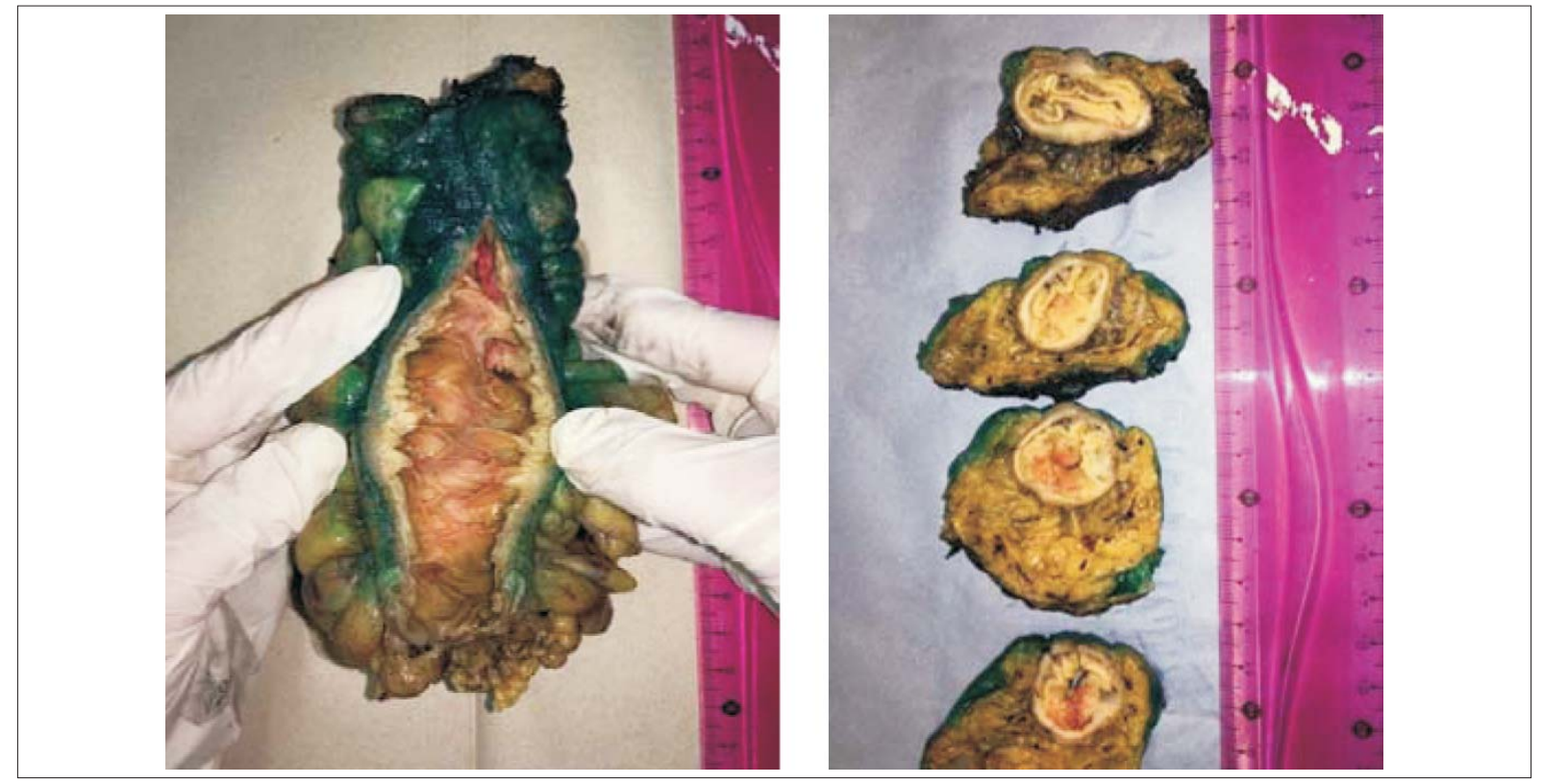

Figura 1. Macroscopía. Foto: Departamento de Anatomía Patológica del Hospital Maciel 


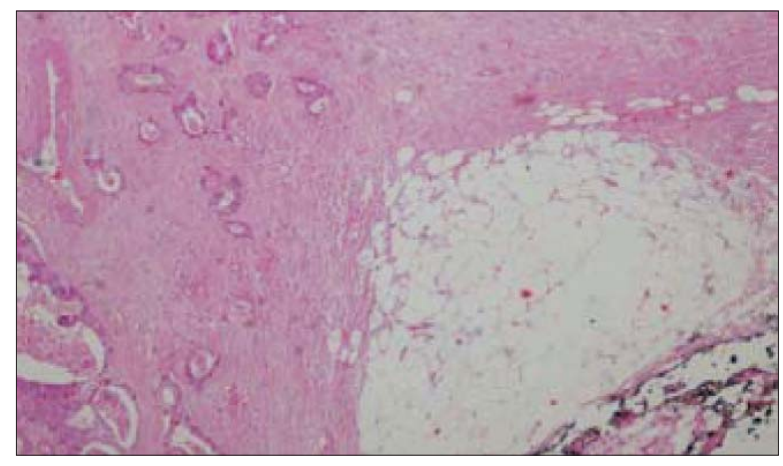

Figura 2. Tumor budding o frente de avance tumoral. Foto: Departamento de Anatomía Patológica del Hospital Maciel

ítem deberá ser consignado sistemáticamente para poder identificar a aquellos pacientes con peor pronóstico (figura 2).

\section{Conclusiones}

La calidad oncológica de la resección de recto depende en gran medida del volumen de cirugías del centro o del equipo quirúrgico actuante, o ambos, hecho que en nuestro medio suele ser una limitante por cuestiones demográficas. Solo teniendo en cuenta este factor (promedio anual de 6-7 resecciones de recto de coordinación en un servicio de cirugía), podemos concluir que la tasa de TME completas $(60 \%)$, así como de linfadenectomías oncológicamente aceptables (50\%), es satisfactoria. El rol del anatomopatólogo en la evaluación de la calidad de la cirugía es indiscutible, por lo que ambos deben trabajar en equipo. Una de las debilidades del trabajo es la heterogeneidad de los informes anatomopatológicos revisados y la falta de información con respecto a variables de interés en algunos de ellos, lo cual se explica por el carácter retrospectivo del estudio, la participación de múltiples técnicos, y a veces por la falta de aporte de datos clínicos precisos por parte del cirujano. Esto resalta aún más la necesidad de que ambas disciplinas trabajen estrechamente, definiendo protocolos de actuación para uniformizar la obtención de todos los datos relevantes.

\section{Abstract}

Introduction: The prognosis of rectal cancer depends, among other factors, on the oncologic quality of the surgical resection, including the correct dissection of the meso-rectum and adequate lymph node dissection

Objectives: to describe the prognostic factors present in rectal cancer resections at the Surgical Clinic 2 of the Maciel Hospital.

Patients and methods: 36 patients underwent coordinated surgery procedures between 2012 and 2016.
Results: meso-rectal dissection was complete in $60 \%$ of the cases, and an acceptable number of lymph nodes were obtained in over $50 \%$ of cases.

Discussion: the quality of oncologic surgery for rectal cancer depends on several factors, being it possible to highlight the number of surgeries per year, the team's experience, patient's factors (sex, body mass index) and disease (low topography, stage, response to neoadyuvancy), among others.

Conclusions: the results obtained can be considered oncologically satisfactory.

\section{Resumo}

Introdução: o prognóstico do câncer de reto depende, entre outros fatores, da qualidade oncológica da ressecção cirúrgica, da correta dissecção mesorretal e do número adequado de gânglios linfáticos obtidos.

Objetivos: descrever os principais fatores que determinam a qualidade da cirurgia de ressecção por câncer de reto na Clínica Quirúrgica 2 do Hospital Maciel.

Pacientes e métodos: 36 pacientes operados em cirurgias eletivas no período 2012-2016.

Resultados: a dissecção mesorretal foi completa em $60 \%$ dos casos, e em mais de $50 \%$ um número aceitável de gânglios linfáticos foi obtido.

Discussão: a qualidade oncológica da cirurgia por câncer de reto depende de múltiplos fatores entre os quais destacamos o volume anual de cirurgias, a experiência da equipe cirúrgica, os fatores do paciente (sexo e índice de massa corporal) e da enfermidade (topografia baja, estádio, resposta à terapia neoadjuvante).

Conclusões: os resultados obtidos podem ser considerados satisfatórios do ponto de vista oncológico.

\section{Bibliografía}

1. Athlin L, Bengtsson NO, Stenling R. Local recurrence and survival after radical resection of rectal carcinoma. Acta Chir Scand 1988; 154(3):225-9.

2. Enker WE, Laffer UT, Block GE. Enhanced survival of patients with colon and rectal cancer is based upon wide anatomic resection. Ann Surg 1979; 190(3):350-60.

3. Heald RJ. A new approach to rectal cancer. Br J Hosp Med 1979; 22(3):277-81

4. Heald RJ, Ryall RD. Recurrence and survival after total mesorectal excision for rectal cancer. Lancet 1986; 1(8496):1479-82.

5. Galler AS, Petrelli NJ, Shakamuri SP. Rectal cancer surgery: a brief history. Surg Oncol 2011; 20(4):223-30.

6. College of American Pathologists. Protocol for the examination of patients with primary carcinoma of the colon and rectum. Washington, DC: CAP, 2016. 
7. Lujan J, Valero G, Hernández Q, Sánchez A, Frutos MD, Parrilla P. Randomized clinical trial comparing laparoscopic and open surgery in patients with rectal cancer. Br J Surg 2009; 96(9):982-9.

8. Kearney DE, Coffey JC. A randomized trial of laparoscopic versus open surgery for rectal cancer. N Engl J Med 2015; 373(2):194

9. Lin HH, Lin JK, Lin CC, Lan YT, Wang HS, Yang SH, et al. Circumferential margin plays an independent impact on the outcome of rectal cancer patients receiving curative total mesorectal excision. Am J Surg 2013; 206(5):771-7.

10. Mezhir JJ, Shia J, Riedel E, Temple LK, Nash GM, Weiser MR, et al. Whole-mount pathologic analysis of rectal cancer following neoadjuvant therapy: implications of margin status on long-term oncologic outcome. Ann Surg 2012; 256(2):274-9.

11. Leonard D, Penninckx F, Fieuws S, Jouret-Mourin A, Sempoux C, Jehaes $\mathbf{C}$, et al. Factors predicting the quality of total mesorectal excision for rectal cancer. Ann Surg 2010; 252(6):982-8.

12. Edge S, Byrd DR, Compton CC, Fritz AG, Greene FL, Trotti A, eds. AJCC Cancer Staging Manual. 7 ed. New York: Springer; 2010.

13. Shin R, Jeong SY, Yoo HY, Park KJ, Heo SC, Kang GH, Kim WH, Park JG. Depth of mesorectal extension has prognostic significance in patients with T3 rectal cancer. Dis Colon Rectum 2012; 55(12):1220-8.

14. Le Voyer TE, Sigurdson ER, Hanlon AL, Mayer RJ, Macdonald JS, Catalano PJ, et al. Colon cancer survival is associated with increasing number of lymph nodes analyzed: a secondary survey of intergroup trial INT-0089. J Clin Oncol 2003; 21(15):2912-9.

15. Mathis KL, Green EM, Sargent DJ, Delaney C, Simmang CL, Nelson H. Surgical quality surrogates do not predict colon cancer survival in the setting of technical credentialing: a report from the prospective COST trial. Ann Surg 2013; 257(1):102-7.

16. Madbouly KM, Abbas KS, Hussein AM. Metastatic lymph node ratio in stage III rectal carcinoma is a valuable prognostic factor even with less than 12 lymph nodes retrieved: a prospective study. Am J Surg 2014; 207(6):824-31.

17. Canessa CE, Badía F, Fierro S, Fiol V, Háyek G. Anatomic study of the lymph nodes of the mesorectum. Dis Colon Rectum 2001; 44(9):1333-6.
18. Tayyab M, Sharma A, Macdonald AW, Gunn J, Hartley JE, Monson JR. Prognostic significance of lymph node ratio in patients undergoing abdominoperineal resection of rectum. Langenbecks Arch Surg 2012; 397(7):1053-7.

19. Nagtegaal ID, Tot T, Jayne DG, McShane P, Nihlberg A, Marshall HC, et al. Lymph nodes, tumor deposits, and TNM: are we getting better? J Clin Oncol 2011; 29(18):2487-92.

20. Shirouzu K, Isomoto H, Kakegawa T. Distal spread of rectal cancer and optimal distal margin of resection for sphincter-preserving surgery. Cancer 1995; 76(3):388-92.

21. Goldstein NS, Soman A, Sacksner J. Disparate surgical margin lengths of colorectal resection specimens between in vivo and in vitro measurements: the effects of surgical resection and formalin fixation on organ shrinkage. Am J Clin Pathol 1999; 111(3):349-51.

22. Birbeck KF, Macklin CP, Tiffin NJ, Parsons W, Dixon MF, Mapstone NP, et al. Rates of circumferential resection margin involvement vary between surgeons and predict outcomes in rectal cancer surgery. Ann Surg 2002; 235(4):449-57.

23. Ryan R, Gibbons D, Hyland JM, Treanor D, White A, Mulcahy HE, et al. Pathological response following long-course neoadjuvant chemoradiotherapy for locally advanced rectal cancer. Histopathology 2005; 47(2):141-6.

24. White M, Foulis AK, Smith G, Horgan PG, Roxburgh CS. The role of S100 staining in the pathological assessment of perineural invasion in rectal cancer. Colorectal Dis 2014; 16(1):71-2.

25. Compton CC. Colorectal carcinoma: diagnostic, prognostic, and molecular features. Mod Pathol 2003; 16(4):376-88.

26. Smith NJ, Barbachano Y, Norman AR, Swift RI, Abulafi AM, Brown G. Prognostic significance of magnetic resonance imaging-detected extramural vascular invasion in rectal cancer. Br J Surg 2008; 95(2):229-36.

27. Prall F. Tumour budding in colorectal carcinoma. Histopathology 2007; 50(1):151-62.

28. Hase K, Shatney C, Johnson D, Trollope M, Vierra M. Prognostic value of tumor "budding" in patients with colorectal cancer. Dis Colon Rectum 1993; 36(7):627-35. 